\section{ARTICLE}

Roger Chun Man Ho is a consultant psychiatrist in the Department of Psychological Medicine, Yong Loo Lin School of Medicine, National University of Singapore. Wendy Cheng is a specialist in emergency medicine in the Department of Emergency Medicine, Queen Elizabeth Hospital, Hong Kong. Anna Nyap Cheng Chua is a research assistant, Department of Psychological Medicine, Yong Loo Lin School of Medicine, National University of Singapore. Anselm Mak is an Assistant Professor of Medicine, Department of Medicine, Yong Loo Lin School of Medicine, National University of Singapore. Correspondence DrRoger Chun Man Ho, Department of Psychological Medicine, National University Hospital, 5 Lower Kent Ridge Road, Singapore 110974. Email:pcmrhcm@nus.edu.sg

\title{
Neuropsychiatric aspects of carbon monoxide poisoning: diagnosis and management
}

\author{
Roger Chun Man Ho, Wendy Cheng, Anna Nyap Cheng Chua \& Anselm Mak
}

\begin{abstract}
SUMMARY
Charcoal-burning has become a common method of attempted suicide in Asia and survivors are often intoxicated with carbon monoxide (CO). Reports of the neuropsychiatric aspects of $\mathrm{CO}$ poisoning date back to 1914 . This article outlines the neuropsychiatric sequelae that have been described secondary to $\mathrm{CO}$ poisoning. The underlying pathophysiology and management strategies, including acute management and cognitive rehabilitation, are discussed as these topics are of relevance to psychiatrists.
\end{abstract}

\section{DECLARATION OF INTEREST}

None.

Carbon monoxide (CO) is a colourless, odourless gas produced as a by-product of incomplete combustion of carbon-containing compounds such as coal, wood, peat, charcoal and petroleum products (Hopkins 2006).

Carbon monoxide poisoning is usually presented with non-specific symptoms similar to flu-like illnesses (Hopkins 2006). It may also present with diverse neurological signs and the level of exposure seems to be the key determinant. The lethal level of carboxyhaemoglobin (COHb) is variable and difficult to predict. There is evidence of oxidation injury following lipid peroxidation in the central nervous system (Thom 1990a). With oxidative stress, nerve conduction becomes impaired and transmission of nerve impulses is delayed as myelin and basic protein are altered (Thom 2004). Neuronal cell death occurs as a result of different mechanisms such as hypoxia (Caine 2000), release of excitatory amino acids (e.g. glutamate) and apoptosis (Piantadosi 1997).

The cause of CO poisoning is heterogeneous, with both accidental and intentional poisoning considered to be common. Victims of CO poisoning are usually young and middle-aged adults (Hopkins 2006). Cobb \& Etzel (1991) reported that intentional CO poisoning is more common in spring and accidental poisoning is more common in winter. Common causes of accidental poisoning include faulty items: furnaces, forklifts, gaspowered saws, water heaters, boats, room heaters, stoves, methylene chloride in paint removers, and fire (Weaver 1999). Carbon monoxide poisoning also occurs when motor vehicles are trapped in the snow with their exhaust pipe buried (Geehr 1989). In the USA, CO poisoning results in approximately 40000 accident and emergency department visits (Hampson 1999) and 800 deaths every year (Piantadosi 1997). The true prevalence may be even higher as some cases have only been discovered during post-mortem examination. Middle-aged adults and those with comorbid cardiac and pulmonary disorders are vulnerable to CO-related mortality (Cobb 1991).

\section{History of $\mathrm{CO}$ poisoning}

\section{In the UK}

Historical records have shown that the Romans used CO to execute criminals (Prockop 2007). In the early 1900s, exposure to fires in closed spaces were common during winter in Great Britain; more recently, faulty gas appliances have often led to accidental CO poisoning (Blumenthal 2001).

Early medical literature describing neuropsychiatric symptoms related to $\mathrm{CO}$ poisoning date back to a case report written by Starkey published in 1914 in the Journal of Mental Science.

\section{'A 56-year-old man was poisoned by $\mathrm{CO}$ gas from a defective stove. He was normal during the first few days. Then he developed progressive stupor, apathy, poor attention and complete disorientation. On neurological examination, he had an unsteady gait, tremulous tongue, exaggerated knee-jerk reflexes and incontinence. After 2 months, his cognitive and neurological abnormalities subsided.'}

During the Second World War, acute and chronic CO poisoning were common, as wood was used as the main source of fuel on the war front (Tvedt 1997). On the home front, Steele $\&$ Hegarty (1950) commented that suicide attempts by coal-gas poisoning were common in England in the 1940s. They reported that a 35-year-old man 
attempted suicide with coal gas, of which CO is the principal constituent. Subsequently, the patient developed complex parieto-occipital syndrome, including Gerstmann's syndrome. In contrast to the patient reported in 1914 , this patient did not recover from his illness (Steele 1950). In the 1960s and 1970s, the switch from coal gas to COfree natural gas caused a dramatic reduction in accidental CO poisoning (Blumenthal 2001).

\section{In the USA}

In the USA, there have been reports of CO poisoning in enclosed vehicle cabins. Griffin et al (2008) reported that a 52-year-old lorry driver felt ill with 'flu-like' symptoms for a few days before being found dead in his secure lorry cabin. He had unknowingly been exposed to a constant level of CO through an exhaust leak in the enclosed cabin. An autopsy report showed that he had developed ischaemic heart failure prior to his death. This was explained by the fact that CO has a high affinity for cardiac myogloblin and such binding causes myocardial depression, hypotension and arrhythmia (Blumenthal 2001).

\section{Other sources of $\mathrm{CO}$}

Carbon monoxide poisoning also occurs without direct inhalation of CO. Methylene chloride (dichloromethane) is an industrial solvent and is found in paint remover. Inhaled or ingested methylene chloride is metabolised to CO by the liver (Ernst 1998; Chang 1999).

\section{The rising trend of $\mathrm{CO}$ poisoning as a suicide method in Asia}

In 1998, a 38-year-old woman took her own life by burning barbecue charcoal in her bedroom in Hong Kong. The sealed room created a CO chamber and the media portrayed this method as a painless way to die, without following the World Health Organization's guidelines on reporting suicide (Liu 2007). This news spread to other Asian countries and created a 'copycat phenomenon'. In Hong Kong, it became the second most common method of suicide 4 years after the first reported case (Liu 2007). In Taiwan, the incidence of charcoalburning suicide rose from 0.22 per 100000 in 1999 to 6.48 per 100000 in 2006 (Pan 2009). In Singapore, we have also witnessed an increase in the incidence of CO intoxication among individuals who attempt suicide in clinical practice.

\section{Assessment of $\mathrm{CO}$ poisoning}

\section{Diagnosis}

The diagnosis of CO poisoning is essentially established by clinical assessment and further confirmed by laboratory investigations. The clinician should enquire about the symptoms associated with CO poisoning in a systematic manner as illustrated in Fig. 1, but there is great variation in clinical presentation. On the one hand, patients may present with subtle physical signs which conceal a significant degree of CO exposure due to delayed neuropsychiatric syndrome. On

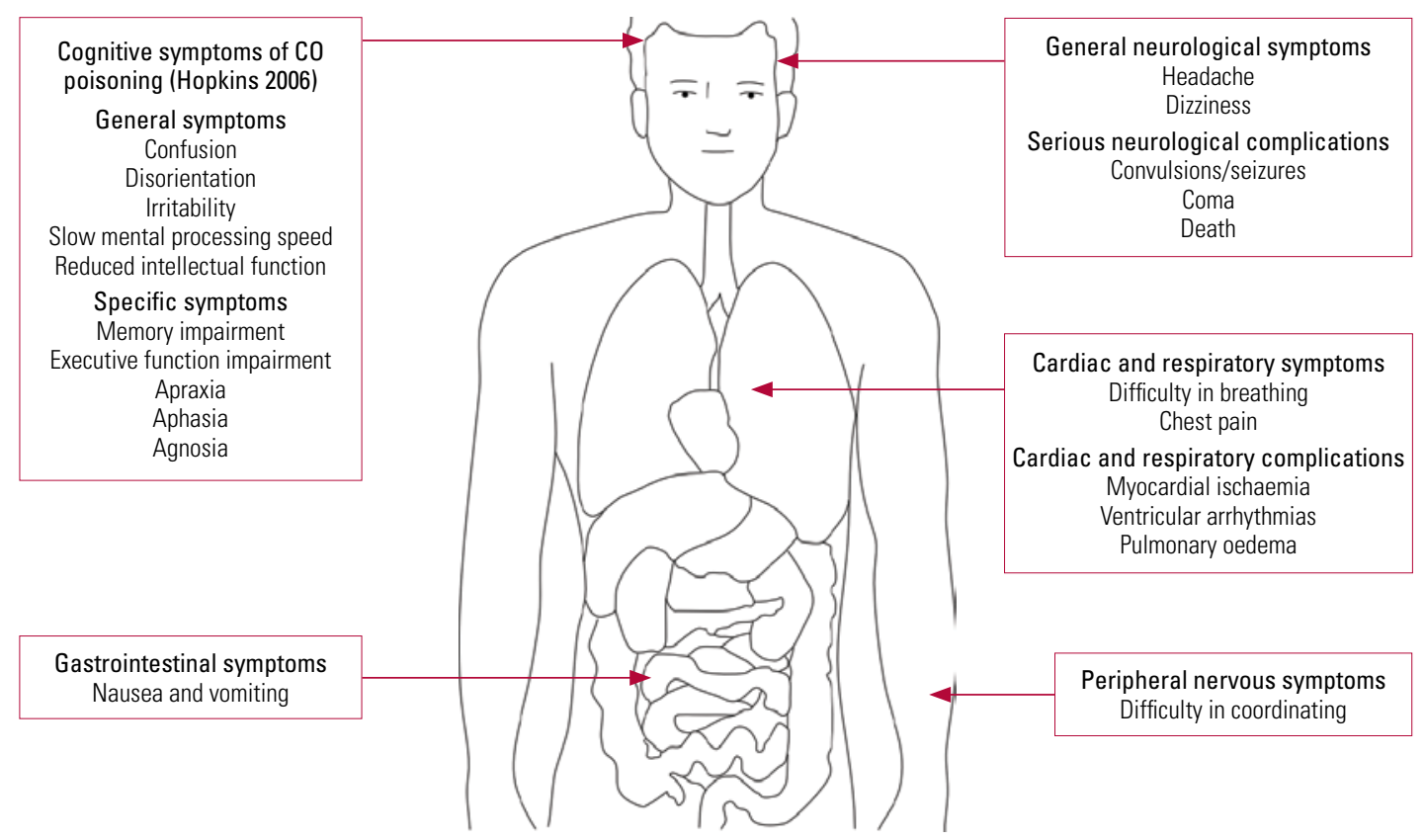


the other hand, some people may already be unconscious when they arrive at hospital. In such cases, individuals have usually sealed off windows to create a closed chamber while the charcoal is burning and have ingested benzodiazepines.

\section{COHb levels}

The level of $\mathrm{COHb}$ is useful to confirm exposure to $\mathrm{CO}$. However, one limitation is that $\mathrm{COHb}$ levels drop rapidly after the person starts to inhale atmospheric air (which contains about 21\% oxygen). Other laboratory tests that may help to determine $\mathrm{COHb}$ levels include arterial blood gases (looking for metabolic acidosis) and blood lactate levels. Informants should be interviewed, as people who have attempted suicide are sometimes reluctant to disclose information. They may have difficulty in recalling the details of the antecedents, the actual suicide attempt and the consequences. An informant close to the patient may also be able to shed valuable light on the usage of faulty devices in cases of accidental poisoning.

\section{Neuropsychiatric syndrome}

The man poisoned by the defective stove in 1914 (Starkey 1914) demonstrated delayed neuropsychiatric syndrome. The onset of the syndrome ranges from 2 to 240 days (Choi 1983) after acute CO exposure. Patients may show subtle abnormalities such as personality changes, mild cognitive deficit to severe dementia and even psychosis (Mimura 1999; Choi 2000). Figure 2 illustrates the delayed and non-delayed neuropsychiatric symptoms after $\mathrm{CO}$ poisoning, with the underlying aetiologies. Clinicians should explore somatic symptoms such as throbbing in the temples, severe headache, generalised weakness, fatigue, sleepiness and dizziness. Physical examination may reveal supporting neurological features such as pseudo-Parkinsonism and evidence of incontinence.

\section{Neuropathological changes following $\mathrm{CO}$ poisoning}

There are a number of reasons why the brain and heart are vulnerable to developing complications after CO poisoning (Prockop 2007). Both the brain and heart have a high metabolic rate, and as mentioned earlier, CO's high affinity for cardiac myogloblin causes myocardial depression, hypotension and arrhythmia (Blumenthal 2001).

Carbon monoxide toxicity may increase the brain's constitutional vulnerability to psychiatric symptoms. Figures 3 and 4 show coronal and sagittal views of the neuroanatomical areas affected by CO poisoning, and the neuropathological changes found in different neuroanatomical areas reported in the literature.

In CO poisoning, neuroimaging changes correspond to physical signs, and one well-reported neuroanatomical area in which these occur is the bilateral globus pallidus (Sawada 1980; Miura 1985; Jones 1994; Silver 1996; Kao 2004). In animal models, the development of low-density lesions in bilateral basal ganglia correlate with the severity of CO exposure through reduction in local blood flow (Song 1983), metabolic acidosis (Kao 2004) and hypotension (Ginsberg 1974; Koehler 1982). The cerebellum shows acute neuropathological changes but the corpus callosum may develop neuropathological changes 2 years after the initial episode of CO poisoning

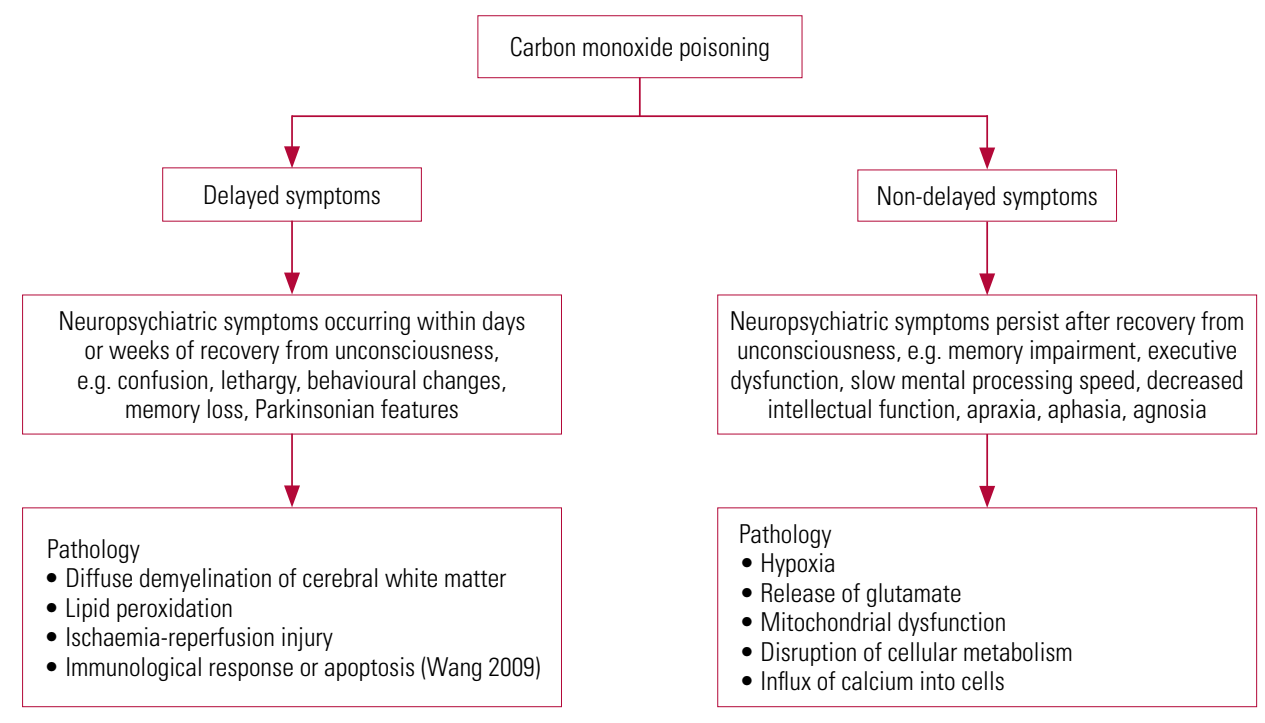




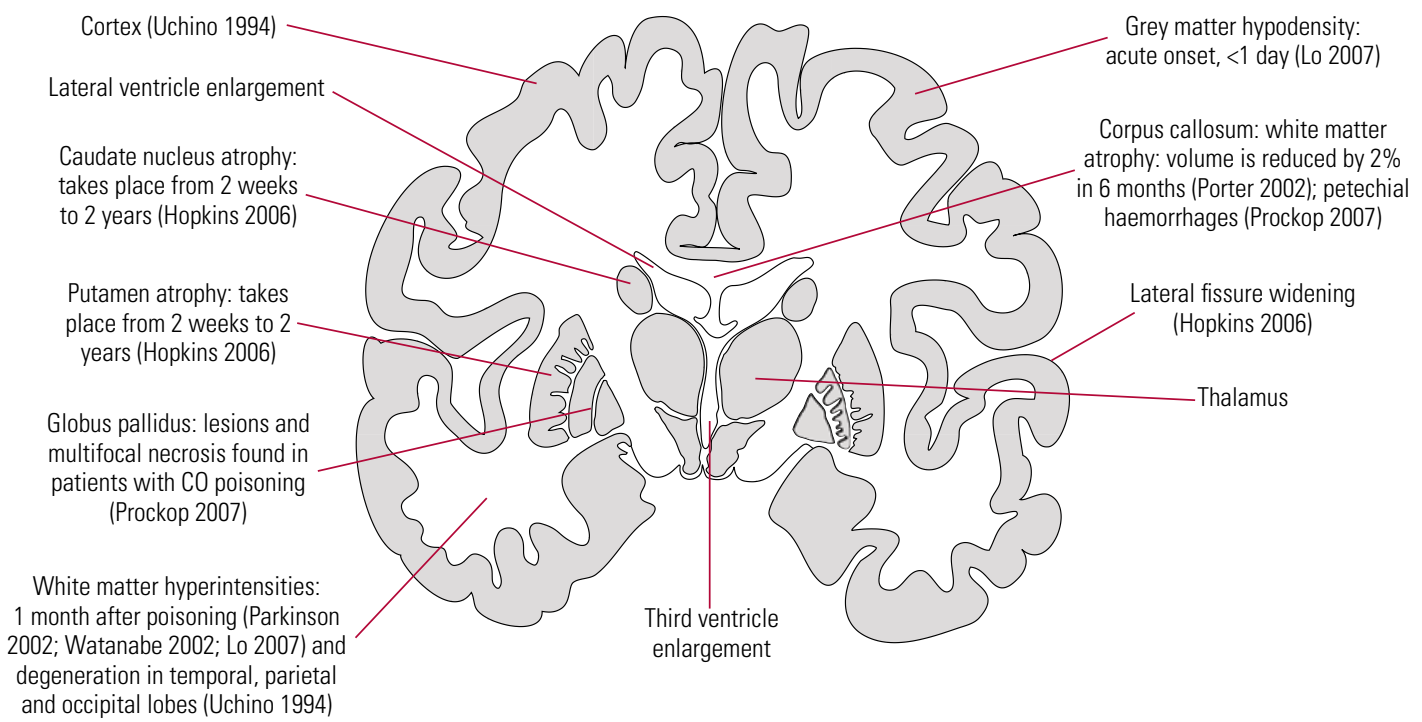

Coronal view of neuroanatomical areas affected by carbon monoxide $(\mathrm{CO})$ poisoning and the associated neuropathological changes with time of onset.

(Lo 2007). On the other hand, both acute and chronic neurological changes occur in the caudate nucleus and putamen (Hopkins 2006).

The onset of neuropathological changes varies from one neuroanatomical area to another and this may underpin the delayed and nondelayed neuropsychiatric symptoms after CO poisoning. Other mechanisms leading to delayed neuropsychiatric sequelae include lipid peroxidation by toxic oxygen species generated by xanthine oxidase (Goldbaum 1975; Thom 1990b,1997,2004; Zhang 1992), ischaemiareperfusion injury and exposure to hyperoxia which may exacerbate the initial oxidative damage (Tomaszewski 1999; Weaver 1999).

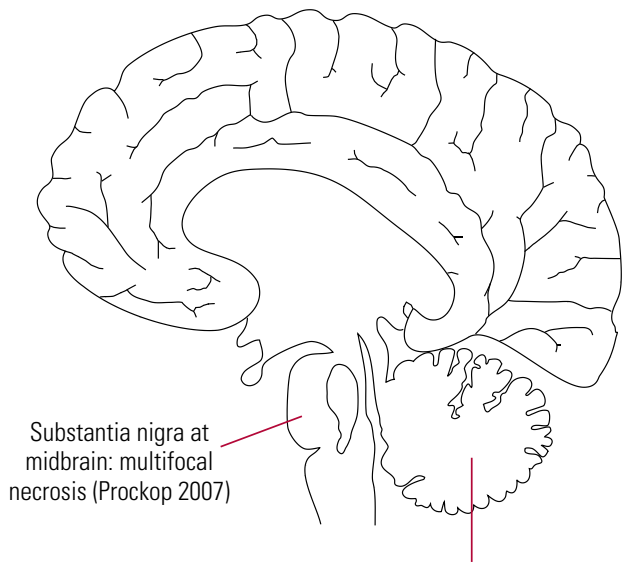

Cerebellum: loss of Purkinje cells (Prockop 2007), associated with acute changes (Lo 2007)

Sagittal view of neuroanatomical areas affected by carbon monoxide poisoning and the associated neuropathological changes.
In the acute setting, computed tomography is helpful to rule out other causes of neurological decompensation (Clardy 2010). For patients with delayed neuropsychiatric sequelae, a magnetic resonance imaging scan may show abnormalities in the globus pallidus and deep white matter (Zagami 1993; Choi 2000; Teksam 2002; Kim 2003; Chu 2004).

\section{Carboxyhaemoglobin}

Carbon monoxide has a 200 times greater affinity for haemoglobin than oxygen. A small concentration of CO can cause significant hypoxia by reducing oxygen release, especially in neuroanatomical areas which require large oxygen consumption such as the basal ganglia (Blumental 2001). Hypoxia may lead to mitochondrial dysfunction and lipid peroxidation, resulting in reversible demyelination. Since the demyelinating action of CO poisoning affects white matter (Prockop 2007) and causes other neuropathological changes in grey matter, cognitive dysfunction associated with CO poisoning may lead to both cortical and subcortical dementia. This gives rise to diverse neuropsychiatric presentations. Figure 5 illustrates the relationship between neuropsychiatric sequelae and $\mathrm{COHb}$ levels.

At baseline, non-smokers' $\mathrm{COHb}$ levels range from 0 to $5 \%$, whereas the baseline $\mathrm{COHb}$ levels of smokers range from 10 to 15\% (Ernst 1998). Baseline $\mathrm{COHb}$ levels are also elevated in people with chronic obstructive pulmonary disease. More specific symptoms may emerge if the $\mathrm{COHb}$ level is greater than $20 \%$, and life-threatening consequences may occur if it is higher than $40 \%$. 


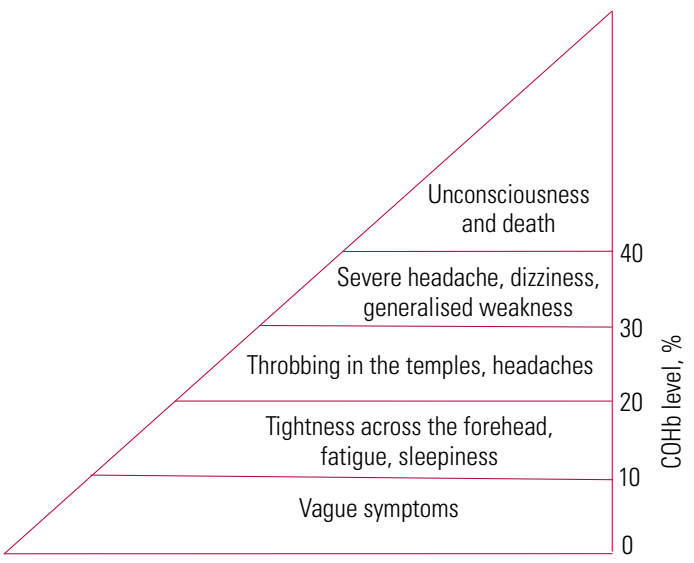

FIG 5 The relationship between neuropsychiatric sequelae after carbon monoxide poisoning and carboxyhaemoglobin (COHb) levels.

Carboxyhaemoglobin levels are poorly correlated with the degree of poisoning and are not predictive of delayed neuropsychiatric sequelae. Levels of $\mathrm{COHb}$ are significantly higher in individuals who die from suicide compared with individuals with accidental CO poisoning (Hopkins 2006), and the blood alcohol level has a positive correlation with $\mathrm{COHb}$ level. Lethal $\mathrm{COHb}$ levels are significantly lower in people with concomitant cardiac or pulmonary diseases.

\section{Other investigations}

A chest radiograph (looking for non-cardiogenic pulmonary oedema), drug levels such as paracetamol and salicylate, blood lactate levels, arterial blood gases (looking for metabolic acidosis) and an electrocardiogram (looking for arrhythmia, changes associated with myocardial ischaemia) should be performed in the accident and emergency department. The presence of metabolic acidosis (Kao 2004) and raised lactate levels (Sokal 1985) correlate well with severity of poisoning.

\section{Baseline and regular neuropsychological assessment}

Neuropsychological assessment should be carried out once the patient's physical condition is stable. Baseline neuropsychological assessment after CO poisoning is certainly useful as it will allow clinicians to measure improvement or deterioration at regular intervals. This will also guide appropriate rehabilitation strategies.

The Carbon Monoxide Neuropsychological Screening Battery (CONSB) was designed specifically for patients with CO poisoning (Messier 1991). This battery consists of six domains assessing general orientation, digit span, trail making, digit symbol, aphasia and block design. Alternatively, neuropsychologists should assess major cognitive functions including retrieval and global memory (Rey Auditory Verbal Learning Test), visuospatial activities (Rey Complex Figure Test), attention and concentration (Wechsler Adult Intelligence Scale), difficulties with abstract thinking (proverb interpretation) and psychomotor speed (Wechsler Adult Intelligence Scale) (Mak 2009).

\section{Predictors for neuropsychiatric sequelae}

One study has looked at the prevalence of cognitive impairment in CO poisoning. Weaver et al (2007) studied 163 patients with CO poisoning but not receiving treatment: $42 \%$ developed neuropsychiatric sequelae. Risk factors for sequelae were loss of consciousness after CO poisoning, being aged 36 years and above, and $\mathrm{COHb}$ levels $\geq 25 \%$. Patients with abnormal neuroimaging findings are more likely to have poorer outcomes but there is no specific neuroimaging marker which indicates prognosis (Sawada 1980; Jones 1994; Silver 1996; Kao 2004).

Lee $\&$ Leung (2008) conducted a similar study with 69 patients after charcoal-burning suicide attempts. The presence of past psychiatric treatment, history of suicide attempt and physical complications were associated with psychiatric comorbidity, whereas loss of consciousness and abnormal blood acidity were associated with medical comorbidity.

\section{Management}

Diagnosis is the most important step - being highly aware that the patient's ill health may be due to $\mathrm{CO}$ exposure. This, in itself, can be of tremendous therapeutic benefit, since it will prompt the paramedics and accident and emergency doctors to start appropriate treatment as early as possible.

The first step of management is stabilisation of the patient's vital status (airway, breathing and circulation). Immediate resuscitation should be performed if the patient is in a critical condition. The second step is assessment of the airway, breathing and circulation. Intubation is indicated in patients in a coma or in those who are severely drowsy. We recommend the immediate use of a $100 \%$ oxygen mask once a patient is suspected of CO poisoning. We also recommend strict bed rest to reduce oxygen demand and consumption. Patients with respiratory distress and decreased level of consciousness should be intubated and ventilated.

\section{Oxygen therapy}

The most common complication of $\mathrm{CO}$ poisoning is hypoxia. Oxygen therapy is effective as most 
symptoms resolve with high flow of oxygen. The half-life of CO is significantly reduced from $300 \mathrm{~min}$ in atmospheric air to $30 \mathrm{~min}$ in $100 \%$ hyperbaric oxygen (Clardy 2010).

There are two types of therapy using 100\% oxygen. In hyperbaric oxygen therapy, the oxygen is at an atmospheric pressure two to three times that of atmospheric pressure at sea level, whereas in normobaric oxygen therapy, the pressure is equivalent to atmospheric pressure at sea level. The main reported benefit of hyperbaric oxygen therapy is its ability to raise the partial pressure of oxygen in arterial blood while decreasing the half-life of $\mathrm{CO}$, thus facilitating its dissociation from haemoglobin. This allows more oxygen to attach to the free-binding sites of haemoglobin, reducing lipid peroxidation and neuropsychiatric sequelae associated with CO poisoning (Prockop 2007). In addition, hyperbaric oxygen offers more benefits to the brain compared with normobaric oxygen by improving energy metabolism and decreasing neutrophil adherence (Stoller 2007). The indications for hyperbaric oxygen treatment include a $\mathrm{COHb}$ level above 25\% (regardless of symptomatology), evidence of ongoing end-organ ischaemia (regardless of $\mathrm{COHb}$ level), profound metabolic acidosis ( $\mathrm{pH}<7.1)$, loss of consciousness and, in pregnant women, a COHb level $>15 \%$ or evidence of fetal distress (Hampson 1995; Ernst 1998; Weaver 1999; Kao 2004). A typical regime involves administering hyperbaric oxygen treatment at 2.5-3.0 atm for about $45 \mathrm{~min}$ in a monoplace chamber.

Despite its potential benefits, hyperbaric oxygen therapy is associated with adverse effects such as reversible myopia, cataracts, tracheobronchial symptoms, self-limited seizures and barotraumas to the middle ear, the cranial sinuses or the lungs. Another limitation is that not all hospitals are equipped with such a chamber. For patients with mild CO poisoning (COHb level $<20 \%$ ), a different regime involving $100 \%$ normobaric oxygen for $6 \mathrm{~h}$ would be appropriate.

\section{Role of donepezil in $\mathrm{CO}$ poisoning}

Donepezil, an acetylcholinesterase inhibitor which is commonly used in the treatment of Alzheimer's disease, has been shown to improve memory in CO poisoning in a case report (Wang 2009). This report evaluated a 60-year-old man with a $\mathrm{COHb}$ level of $11.2 \%$. He developed neuropsychological impairment after 15 days. Donepezil (10 mg/day) treatment showed improvement in the Mini-Mental State Examination after 2 months. Nevertheless, the overall benefits of donepezil remain unknown. A large, multicentre investigation of the therapeutic effects of donepezil or other acetylcholinesterase inhibitors in $\mathrm{CO}$ poisoning is warranted.

\section{Role of cognitive rehabilitation in CO poisoning}

There is no well-established rehabilitation programme for patients with cognitive impairment associated with CO poisoning. A suitable programme will have to be created from existing strategies for other neuropsychiatric disorders such as head injury:

- individualised treatment with particular focus on the patient's background, addressing the emotional responses after CO poisoning and developing coping skills for long-term adjustment (Mateer 2005);

- components such as psychoeducation on the neuropsychiatric sequelae of CO poisoning, identification of behavioural deficits (Mangaoang 2007), reinforcing previously learned behaviour, establishing compensatory mechanisms for cognitive deficits (Bergquist 1997), and applying the strategies learnt to suit the patient's need through repeated practice;

- comparing baseline and post-rehabilitation neuropsychological assessments, helping to monitor treatment progress and giving feedback to the patient and caregivers.

\section{Conclusions}

Carbon monoxide poisoning has been reported throughout medical history and its presentation has changed from accidental poisoning due to faulty stoves in the UK almost a century ago to the recent intentional poisoning by individuals who attempt suicide in the Far East. Analyses of case series and advances in imaging techniques have helped clinicians understand the neuropathology of CO poisoning. There is great variation in the onset and clinical presentation as $\mathrm{CO}$ poisoning affects both cortical and subcortical brain regions.

All patients who are suspected of having CO poisoning should be given 100\% oxygen via a face mask in the accident and emergency department. It should now be clear that the management of CO poisoning does not stop at acute treatment but continues with personalised long-term management in a multidisciplinary setting. All patients should undergo baseline and follow-up neuropsychological assessment. Cognitive rehabilitation targeted at maintaining residual cognitive functions and developing compensatory strategies will be beneficial. Further studies will be required to investigate the effects of acetylcholinesterase inhibitors on cognitive impairment after CO poisoning. 


\begin{tabular}{l} 
MCQ answers \\
$1 \mathrm{~d} \quad 2 \mathrm{e} \quad 3 \mathrm{c} \quad 4 \mathrm{c}$ \\
\hline $\mathrm{e}$
\end{tabular}

\section{References}

Bergquist TF, Malec JF (1997) Psychology: current practice and training issues in treatment of cognitive dysfunction. Neurorehabilitation 45 : 112-29.

Blumenthal I (2001) Carbon monoxide poisoning. Journal of Royal Society of Medicine 94: 270-2.

Caine D, Watson JD (2000) Neuropsychological and neuropathological sequelae of cerebral anoxia: a critical review. Journal of the International Neuropsychological Society 6: 86-99.

Chang YL, Yang CC, Deng JF, et al (1999) Diverse manifestations of oral methylene chloride poisoning: report of 6 cases. Journal of Toxicology Clinical Toxicology 37: 497.

Choi IS (1983) Delayed neurologic sequelae in carbon monoxide intoxication. Archives of Neurology 40: 433-5.

Choi $S$ (2000) Delayed neurological sequelae in carbon monoxide intoxication. Archives of Neurology 57: 1214-8.

Chu K, Jung KH, Kim HJ, et al (2004) Diffusion-weighted MRI and 99mTcHMPAO SPECT in delayed relapsing type of carbon monoxide poisoning: evidence of delayed cytotoxic edema. European Neurology 51: 98

Clardy PF, Manaker S, Perry H, et al (2010) Carbon monoxide poisoning. UpToDate (online journal: http:// http://www.uptodate.com).

Cobb N, Etzel RA (1991) Unintentional carbon monoxide-related deaths in the United States, 1979 through 1988. Journal of the American Medical Association 266: 659-63.

Ernst A, Zibrak JD (1998) Carbon monoxide poisoning. New England Journal of Medicine 339: 1603

Geehr EC, Salluzzo R, Bosco S, et al (1989) Emergency health impact of a severe storm. American Journal of Emergency Medicine 7: 598-604.

Ginsberg MD, Myers RE, McDonagh RF (1974) Experimental carbon monoxide encephalopathy in the primate: II. Clinical aspects, neuropathology, and physiologic correlation. Archive of Neurology 30 : 209-16

Goldbaum LR, Ramirez RG, Absalon KB (1975) What is the mechanism of carbon monoxide toxicity? Aviation, Space, and Environmental Medicine 46: 1289.

Griffin SM, Ward MK, Terrell AR, et al (2008) Diesel fumes do kill: a case of fatal carbon monoxide poisoning directly attributed to diesel fuel exhaust with a 10-year retrospective case and literature review. American Academy of Forensic Sciences 53: 1206-11.

Hampson NB, Dunford RG, Kramer CC, et al (1995) Selection criteria utilized for hyperbaric oxygen treatment of carbon monoxide poisoning. Journal of Emergency Medicine 13: 227.

Hampson NB (1999) Hyperbaric Oxygen Therapy: 1999 Committee Report. Undersea and Hyperbaric Medical Society.

Hopkins R0, Woon FLM (2006) Neuroimaging, cognitive, and neurobehavioural outcomes following carbon monoxide poisoning. Behavioural and Cognitive Neuroscience Reviews 5: 144-55.

Jones JS, Lagasse J, Zimmerman G (1994) Computerized tomographic findings after acute carbon monoxide poisoning. American Journal of Emergency Medicine 12: 448-51.

Kao LW, Nanagas KA (2004) Carbon monoxide poisoning. Emergency Medicine Clinics of North America 22: 985.

Kim JH, Chang KH, Song IC, et al (2003) Delayed encephalopathy of acute carbon monoxide intoxication: diffusivity of cerebral white matter lesions. American Journal of Neuroradiology 24: 1592

Koehler RC, Jones Jr MD, Traystman RJ (1982) Cerebral circulatory response to carbon monoxide and hypoxic hypoxia in the lamb. American Journal of Physiology 243: 27-32

Lee E, Leung CM (2008) Clinical predictors of psychiatric and medical morbidities of charcoal-burning suicide attempt in Hong Kong. General Hospital Psychiatry 30: 561-3.

Liu KY, Beautrais A, Caine E, et al (2007) Charcoal burning suicides in Hong Kong and urban Taiwan: an illustration of the impact of a novel suicide method on overall regional rates. Journal of Epidemiology and Community Health 61: 248-53.
Lo C-P, Chen S-Y, Lee K-W, et al (2007) Brain injury after acute carbon monoxide poisoning: early and late complications. Neuroradiology 189 : W205-11

Mak A, Ho RCM, Lau CS (2009) Clinical implications of neuropsychiatric systemic lupus erythematosus. Advances in Psychiatric Treatment 15: 451-8.

Mangaoang MA, Lucey JV (2007) Cognitive rehabilitation: assessment and treatment of persistent memory impairments following ECT. Advances in Psychiatric Treatment 13: 90-100.

Mannaioni PF, Vannacci A, Masini E (2006) Carbon monoxide: the bad and the good side of the coin, from neuronal death to anti-inflammatory activity. Inflammation Research 55: 261-73

Mateer CA, Sira CS, O'Connell ME (2005) Putting Humpty Dumpty together again: the importance of integrating cognitive and emotional interventions. Journal of Head Trauma and Rehabilitation 20: 62-75.

Messier LD, Myers RA (1991) A neuropsychological screening battery for emergency assessment of carbon-monoxide-poisoned patients. Journal of Clinical Psychology 47: 675-84.

Mimura K, Harada M, Sumiyoshi S, et al (1999) Long-term follow-up study on sequelae of carbon monoxide poisoning: a serial investigation 33 years after poisoning. Seishin Shinkeigaku Zasshi 101: 592-618.

Miura T, Mitomo M, Kawai R, et al (1985) CT of the brain in acute carbon monoxide intoxication: characteristic features and prognosis. American Journal of Neuroradiology 6: 739-42

Pan Y-J, Liao S-C, Lee M-B (2009) Suicide by charcoal burning in Taiwan, 1995-2006. Journal of Affective Disorders 120: 254-7.

Parkinson RB, Hopkins RO, Cleavinger HB, et al (2002) White matter hyperintensities and neuropsychological outcome following carbon monoxide poisoning. Neurology 58: 1525-32.

Piantadosi CA, Zhang J, Levin ED, et al (1997) Apoptosis and delayed neuronal damage after carbon monoxide poisoning in the rat. Experimental Neurology 147: 103-14.

Porter SS, Hopkins RO, Weaver LK, et al (2002) Corpus callosum atrophy and neuropsychological outcome following carbon monoxide poisoning. Archives of Clinical Neuropsychology 17: 195-204.

Prockop LD, Chichkova RI (2007) Carbon monoxide intoxication: an updated review. Journal of Neurological Sciences 262: 122-30.

Sawada Y, Takahashi M, Ohashi N, et al (1980) Computerised tomography as an indication of long-term outcome after acute carbon monoxide poisoning. Lancet 1: 783-4.

Silver DA, Cross M, Fox B, et al (1996) Computerized tomography of the brain in acute carbon monoxide poisoning. Clinical Radiology 51: 480-3.

Sokal JA, Kralkowska E (1985) The relationship between exposure duration, carboxylhaemoglobin, blood glucose, pyruvate and lactate and the severity of intoxication in 39 cases of acute carbon monoxide poisoning in man. Achive of Toxicology 57: 196-9.

Song SY, Okeda R, Funata N, et al (1983) An experimental study of pathogenesis of the selective lesion of the globus pallidus in acute carbon monoxide poisoning in cats. Acta Neuropathologica 61: 232-8.

Starkey W (1914) Carbon monoxide poisoning. Journal of Mental Science 60: 120

Steele GDF, Hegarty AB (1950) Parieto-occipital syndrome following carbon monoxide poisoning. Journal of Mental Science 96: 1015-23.

Stoller KP (2007) Hyperbaric oxygen and carbon monoxide poisoning: a critical review. Neurological Research 29: 146-55.

Teksam M, Casey SO, Michel E, et al (2002) Diffusion-weighted MR imaging findings in carbon monoxide poisoning. Neuroradiology 44: 109.

Tomaszewski C (1999) Carbon monoxide poisoning. Early awareness and intervention can save lives. Postgraduate Medicine 105: 39.

Thom SR (1990a) Antagonism of carbon monoxide mediated brain lipid peroxidation by hyperbaric oxygen. Toxicology and Applied Pharmacology 105: 340-4.

Thom SR (1990b) Carbon monoxide-mediated brain lipid peroxidation in the rat. Journal of Applied Physiology 68: 997. 
Thom SR, Xu YA, Ischiropoulos H (1997) Vascular endothelial cells generate peroxynitrite in response to carbon monoxide exposure. Chemical Research in Toxicology 10: 1023

Thom SR, Bhopale VM, Fisher D, et al (2004) Delayed neuropathy after carbon monoxide poisoning is immune-mediated. Proceedings of the National Academy of Sciences of the United States of America 101: 13660-5.

Tvedt B, Kjuus H (1997) Chronic CO poisoning. Use of generator gas during the Second World War and recent research. Tidsskr Nor Laegoforen 117 : $2454-7$

Uchino A, Hasuo K, Shida K, et al (1994) MRI of the brain in chronic carbon monoxide poisoning. Neuroradiology 36: 399-401.

Wang P, Zeng T, Chi Z-F (2009) Recovery of cognitive dysfunction in a case of delayed encephalopathy of carbon monoxide poisoning after treatment with donepezil hydrochloride. Neurology India 57: 481-2.
Watanabe N, Nohara S, Matsuda H, et al (2002) Statistical parametric mapping in brain single photon computed emission topography after carbon monoxide intoxication. Nuclear Medicine Communications 23: $355-66$

Weaver LK (1999) Carbon monoxide poisoning. Critical Care Clinics 15 297-317.

Weaver LK, Valentine KJ, Hopkins RO (2007) Carbon monoxide poisoning risk factors for cognitive sequelae and the role of hyperbaric oxygen. American Journal of Respiratory and Critical Care Medicine 176: 491-7.

Zhang J, Piantadosi CA (1992) Mitochondrial oxidative stress after carbon monoxide hypoxia in the rat brain. Journal of Clinical Investigation 90: 1193

Zagami AS, Lethlean AK, Mellick R (1993) Delayed neurological deterioration following carbon monoxide poisoning: MRI findings. Journal of Neurology 240: 113
MCOs

Select the single best option for each question stem

1 A 20-year-old man murdered his girlfriend and attempted suicide by connecting the exhaust pipe into his car. He was found by a passerby and sent to accident and emergency (A\&E) by ambulance. You suspect that he may be suffering from $\mathrm{CO}$ poisoning. The following signs and symptoms are commonly found in people after $\mathrm{CO}$ poisoning except for:

a confusion

b memory impairment

c respiratory distress

$\mathrm{d}$ abdominal distension

e vomiting.

2 After you have assessed the patient, you need to advise the A\&E doctors, who are not familiar with the management of $\mathrm{CO}$ poisoning. The following advice is appropriate except for:

a immediate administration of $100 \%$ of oxygen via face mask is recommended

b blood investigations should include $\mathrm{COHb}$ level, blood lactate levels and arterial blood gases

c electrocardiogram is necessary as the cardiovascular system is usually affected in CO poisoning.

$\mathrm{d}$ a chest $\mathrm{X}$-ray is recommended

e immediate consultation for a renal physician to arrange urgent dialysis to remove the $\mathrm{CO}$ from the haemoglobin
3 The laboratory has returned the $\mathrm{COHb}$ leve results. The A\&E doctors want to consult you on the likely neuropsychiatric sequelae in relation to the $\mathrm{COHb}$ level. Which of the following statements is incorrect?

a patients usually present with vague symptoms if the $\mathrm{COHb}$ level is less than $10 \%$

b patients usually present with tightness across the forehead, fatigue and sleepiness if the $\mathrm{COHb}$ level is between 10 and $20 \%$

c the $\mathrm{COHb}$ level is expected to be lower in people who have died by suicide than those with accidental poisoning

d patients usually present with severe headaches, dizziness and generalised weakness when the $\mathrm{COHb}$ level is between 30 and $40 \%$

e patients may become unconscious when the $\mathrm{COHb}$ level is above $40 \%$

4 The patient has been stabilised on the medical ward. The consultant physician has asked you to review the patient and give your professional opinion. Which of the following actions is inappropriate?

a obtaining the underlying reason and consequences of the suicide attempt from both the patient and informants

b exploring his state of mind when the homicide took place and assessing him for current feelings of guilt

c transferring him to police custody once he is free from confusion or cognitive impairment

$\mathrm{d}$ administering hyperbaric oxygen treatment if the facility is available

e regular monitoring of $\mathrm{COHb}$ levels.
5 The patient develops neuropsychiatric complications. His sister would like to discuss with you the long-term management of his case. Which of the following statements would be wrong?

a baseline and follow-up neuropsychological assessments are necessary in this case

$\mathrm{b}$ an assessment of his fitness to plead will be necessary before he appears in court

c cognitive rehabilitation to develop compensatory strategies should be helpful

d on admission, his $\mathrm{COHb}$ level was $35 \%$, putting him at risk of developing neuropsychiatric sequelae

e acetylcholinesterase inhibitors have a strong evidence base in treating cognitive impairment after $\mathrm{CO}$ poisoning. 